\title{
Impact of Plasma Thermal Transients on the design of the EU DEMO first wall protection
}

Francesco Maviglia ${ }^{\mathrm{a}, \mathrm{b},{ }^{*}, \text { Christian Bachmann }}{ }^{\mathrm{a}}$, Gianfranco Federici ${ }^{\mathrm{a}}$, Thomas Franke ${ }^{\mathrm{a}, \mathrm{c}}$, Mattia Siccinio $^{\mathrm{a}, \mathrm{c}}$, Christian Vorpahl ${ }^{\mathrm{a}}$, Raffaele Albanese ${ }^{\mathrm{d}}$, Roberto Ambrosino ${ }^{\mathrm{d}}$, Emiliano Fable ${ }^{\mathrm{c}}$, Mehdi Firdaous $^{\mathrm{e}}$, Jonathan Gerardin ${ }^{\mathrm{e}}$, Vincenzo Paolo Loschiavo ${ }^{\mathrm{d}}$, Massimiliano Mattei ${ }^{\mathrm{d}}$, Francesco Palermo ${ }^{\mathrm{c}}$, Maria Lorena Richiusa ${ }^{\mathrm{f}}$, Fabio Villone ${ }^{\mathrm{d}}$, Zsolt Vizvary ${ }^{\mathrm{f}}$

\author{
${ }^{a}$ EUROfusion - Programme Management Unit, Boltzmannstrasse 2, 85748 Garching, Germany \\ ${ }^{b}$ Associazione EURATOM-ENEA sulla Fusione, C.R. Frascati, C.P. 65-00044 Frascati, Rome, Italy \\ ${ }^{c}$ Max-Planck-Institut für Plasmaphysik, Garching, Germany \\ ${ }^{d}$ Consorzio CREATE, Univ. Napoli Federico II - DIETI, 80125 Napoli, Italy \\ ${ }^{e}$ CEA, F-13108 St Paul-Lez-Durance, France \\ ${ }^{f} C C F E$, Culham Science Centre, Abingdon, Oxon, OX14 3DB, UK \\ *Corresponding author: francesco.maviglia@euro-fusion.org
}

\begin{abstract}
The protection of the EU-DEMO first wall (FW) during plasma transients represents one of the main challenges of the current pre-concept design phase. While the present DEMO FW design heat load capability is of the order of $\approx 1-2 \mathrm{MW} / \mathrm{m}^{2}$ in steady state, this limit is overcome during plasma transients for both normal and off-normal events leading to a plasma-wall contact. A strategy to protect the FW is being developed, considering the inclusion of discrete limiters, designed also to maintain the integrity of their cooling system during transients. The present investigations include electromagnetic modelling and plasma simulations on a list of critical transient events. The plasma equilibria are designed to ensure that the plasma impact, in case of loss of plasma control, is located, when possible, close to maintenance ports, to allow for replacement of the limiters. Charged particles and radiation heat load calculations are performed to evaluate the surface design and the required number of limiters. Finally, simplified thermal analyses are run to verify the integrity of the limiter plasma-facing components, and propose their design.
\end{abstract}

Keywords: DEMO, Plasma transients, first wall load, electromagnetic simulations, plasma scenario optimization, discrete limiters.

\section{Introduction}

The design, performance and feasibility of wall protection limiters during plasma transients, was selected as one of the Key Design Integration Issues [1] (KDII) within the present European DEMO pre-concept design phase. The present DEMO breeding blanket (BB) first wall $(\mathrm{FW})$ has a steady-state heat load capability of no more than $\approx 1-2 \mathrm{MW} / \mathrm{m}^{2}$ [2], for both helium and watercooled concepts. This is due to the DEMO specific requirements to use high neutron irradiation resistant materials, such as EUROfer, to have high coolant temperature, for an efficient energy conversion, and to maximize the tritium breeding ratio (TBR) [3, 4]. The strategy being developed for protecting the DEMO FW from plasma transients includes discrete high heat flux limiters. DEMO scenarios and geometry have been developed in recent years to comply with the $1 \mathrm{MW} / \mathrm{m}^{2}$ heat flux limit on the whole BB FW during the flat top steady state phases [4], including radiation and charged particle loads. Such limit would be exceed should the plasma become in contact with the BB FW during plasma transients, e.g. plasma ramp-up/down, and off-normal events, e.g. Upward or Downward Vertical Displacement Events (U/D-VDEs), disruptions, and unforeseen H-L transitions. This paper presents the current status of the developed FW protection strategy. In the first part are presented the 2D/3D electromagnetic (EM) simulations of a list of plasma transients, as complete as currently available, with the aim to predict all the possible poloidal locations of plasma-wall contact. Plasma scenario and machine geometry optimization are also presented, with the aim of designing such contact locations in areas where it is expected that a limiter can be more easily maintainable. These simulations are then used to evaluate the heat flux on the FW and limiters due to radiation and charged particles. The final aim is to be able to protect the FW from damages, while minimizing the number of limiters, to minimize the impact on TBR and reduce cost/complexity. Finally, the resulting heat flux loads and deposition times are used to run simplified thermohydraulic simulations to evaluate different limiter designs for different functions that the limiters have to perform, depending on the type of transients. The aim is to evaluate the thermal behavior of the plasma facing components (PFC) in order to designing them to maintain the integrity of their cooling system, and estimate the number events that the limiters can withstand in the different cases.

\section{DEMO 2D/3D electromagnetic model studies}

The EU-DEMO plasma scenarios and machine geometry are developed and optimized using different EM codes, during the present pre-conceptual design phase. These are the 2D codes CREATE NL [5] and MAXFEA [6], which are benchmarked with each other, and with experimental data, and the 3D code CARMA0NL [7], which aims at more detailed analysis of 3D features, such as non-toroidal continuous electrical conductive structures (e.g. the $\mathrm{BB}$ modules, and the VV ports). A recent approach used for DEMO is the modelling of the effect of the mentioned 3D features by using equivalent $2 \mathrm{D}$ passive structures. This approach 
uses 3D linearized models from the CARMA0NL [8] and includes in the CREATE-NL equivalent conductive structures, in blue in Fig. 1, modelling the BB (previously not considered) and the VV 3D features, whose resistance and inductance, also of the $\mathrm{VV}$, are tuned to best fit the input/output dynamic behavior of the 3D model.
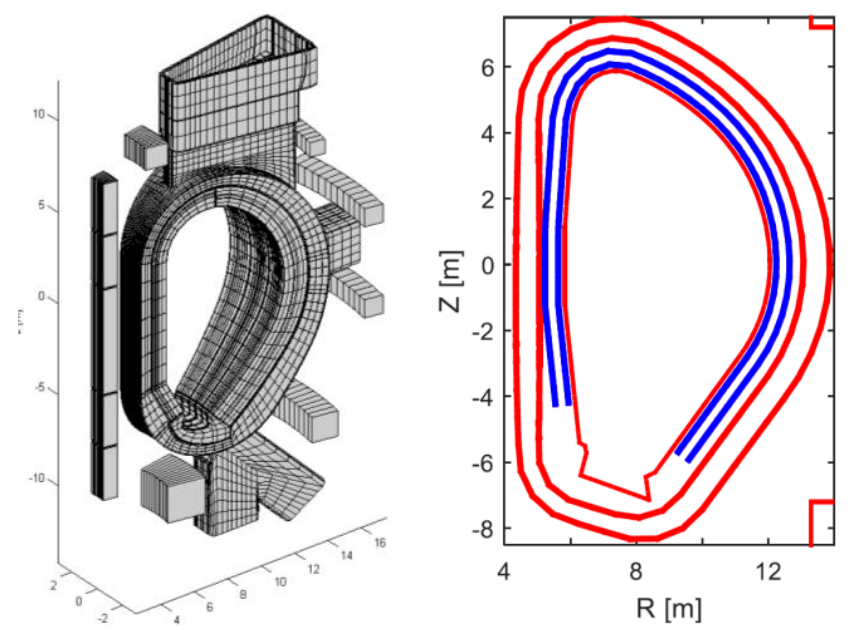

Fig. 1 Comparison of the 3D model (on the left) including nonaxisymmetric $\mathrm{BB}$, and $\mathrm{VV}$ ports, with the equivalent $2 \mathrm{D}$ model (on the right) which includes additional conductive structures (in blue) tuned to best fit the dynamic behavior of the former in a frequency range of interest.

The results, for which the details are described in [9], show a very good agreement of the inputs/outputs transfer function, in the frequency range of interest, by matching the Bode diagrams for the radial and vertical fields produced by the eddy currents at the center of the machine, to the radial and vertical fields generated by the external poloidal filed coils. The 2D model developed, with the $3 \mathrm{D}$ correction, is used to increase the precision of the dynamic simulations while maintaining the lower computational requirements, hence allowing a broader span of simulation capability.

\section{DEMO plasma scenario optimization}

Recent studies [10] have shown that it is possible to predict the plasma-wall contact point in case of VDE, by evaluating the plasma magnetic flux map in nominal conditions, i.e. before the disruption, and knowing the time constants of the conductive structures surrounding the plasma and the disruption duration. This prediction capability is used in this paper to support the development of a wall protection strategy from plasma transients. Based on the present DEMO baseline scenarios [11], a new set of Start and End Of Flattop (SOF/EOF) equilibria have been developed, in which a scan of the plasma triangularity is performed, while keeping all the other plasma parameters unchanged. Previously the plasmawall contact points were located at the top of the inboard $\mathrm{BB}$ in the area of the $2^{\text {nd }}$ null for all U-VDE phases described in detail in the next paragraph and shown in Fig. 2. The new equilibria have new predicted plasma-wall contact points, in case of Upward-VDE. These new points have been designed to be located in the location of the upper vertical ports [12]. The new scenario was obtained by performing a sensitivity scan on the position of the upper inactive x-point, which is rotated clockwise, such as to reduce the total triangularity at $95 \%$ of the boundary flux, $\delta_{95 \%}$, from 0.33 down to 0.25 . This is evaluated to be an acceptable compromise on the plasma performances, also considering the present uncertainties regarding the influence of such parameters on the new DEMO scenario studies [13].
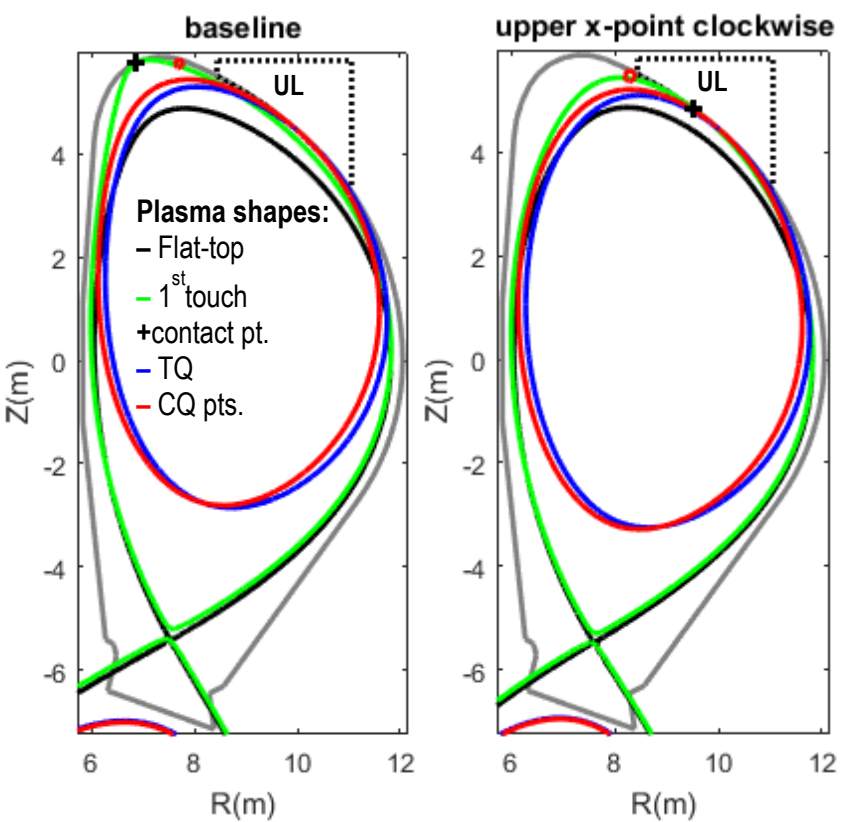

Fig. 2 Comparison of Plasma U-VDE simulations of DEMO baseline (left) and optimized (right) configurations, including: Nominal EOF (black), first plasma-wall touch (green, with black + symbol as contact point), TQ (blue) and CQ points (red, initial and final) phases, upper port region (dotted area).

\section{DEMO plasma transient database}

A list of plasma transients is being compiled within the DEMO wall protection strategy activities. While the intention is to have a list as complete as possible, according to the present knowledge, such list is intended to be as a work in progress for the years to come, and hence it will be updated once new events, scenarios, or DEMO designs are available. For the purpose of this paper such list is used to predict all the poloidal location on the FW for the events that lead the plasma to touch it. This list is divided in the categories hereafter listed.

\subsection{Normal events}

These events must happen at every discharge, and are due to limitations of the plasma shape controller when the plasma has low current compared to the one in the passive structures:

Plasma current ramp-up. The present simulated scenario of this phase, as described in [4], indicates a maximum ramp rate in the range $0.1-0.2 \mathrm{MA} / \mathrm{s}$, and an earliest possible transition from limited (with the plasma last closed surface touching the wall) to the diverted configuration (with the plasma not in contact with the wall and the presence of a magnetic field null, named x-point, inside the chamber), in the range 3.5-6MA. The combination of ramp-rates and configuration transition currents, leads to a plasma-wall contact from $17.5 \mathrm{~s}$ to $60 \mathrm{~s}$, 
respectively in the best and worst combination. For the total power in the scrape-off-layer (SOL) during the limited phase $\left(P_{\text {sol, lim }}\right)$, the same ITER hypothesis [14] was used:

$$
P_{\text {sol, } \lim }(M W)=I_{p}(M A),
$$

with $I_{p}=$ plasma current. The near SOL e-folding length $\left(\lambda_{q}\right)$ prediction for DEMO for the outboard limiter configuration is $\approx 5-6 \mathrm{~mm}$, as described in [15].
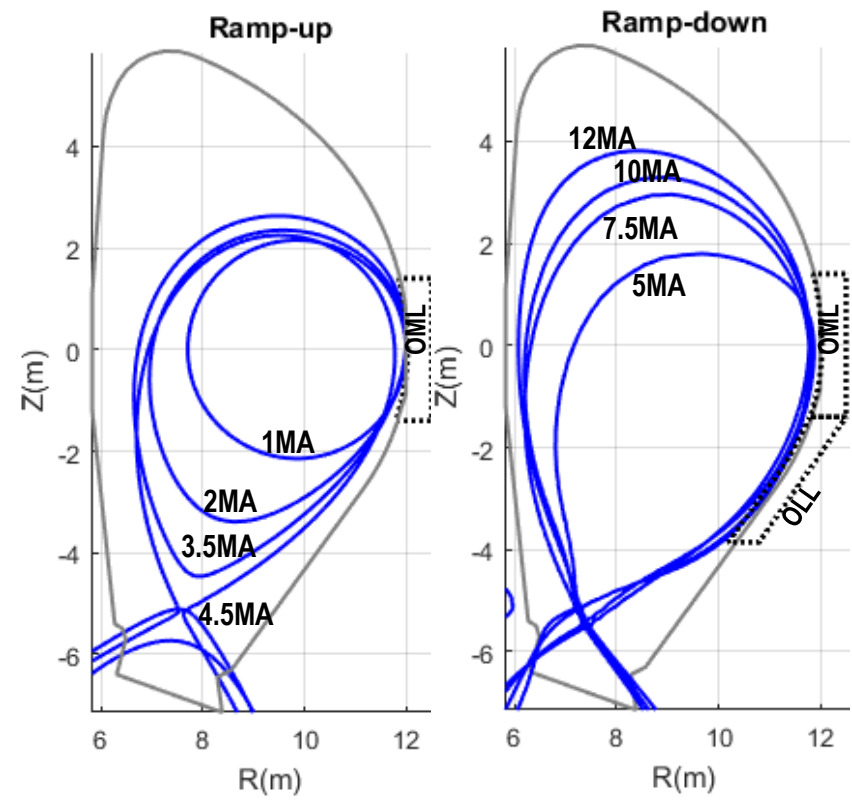

Fig. 3 Example of plasma scenarios for plasma current rampup (left) and ramp-down (right), developed on the outer midplane.

Plasma current ramp-down. A recent simulation on this phase indicates that it is possible to maintain the diverted configuration, with the plasma not touching the wall, until the plasma current value is reduced down to 5-7MA. By combining this values with the ramp-down rate of 0.1 $0.2 \mathrm{MA} / \mathrm{s}$ (as for the ramp-up), it is possible to estimate a plasma wall contact time in the range from $25 \mathrm{~s}$ to $75 \mathrm{~s}$, respectively in the best and worst case. Similarly to the ramp up, the same eq. (1) and same e-folding length hypotheses are used, leading to $P_{\text {sol, } \lim }(\mathrm{MW})=5-7.5 \mathrm{MW}$, and $\lambda_{q} \approx 5-6 \mathrm{~mm}$.

The ramp-up and down phases have been simulated on an outboard mid-plane limiter, which is thought to be preferable for a better maintainability of the limiter, and to keep a distinct function from a protection limiter from off-normal events. In alternative the possibility to ramp the plasma on an inboard mid-plane limiter is being developed in parallel. An image of the developed rampup and down scenarios is shown in Fig. 3.

\subsection{Off-normal events}

One of the main DEMO requirements is the minimization of plasma perturbations and instabilities that may lead to disruptions, by looking for a suitable EUDEMO scenario, as described in [13]. The scope of this paper is to elaborate a strategy for the FW protection in case that such occurrence happens. These events may occur, for instance, in case of technical issues with the plasma control systems, or due to plasma perturbations beyond controllable limits, or impurities entering the plasma above the stability level. Hereafter will follow the list of events considered, some of which are shown in Fig. 5:

Plasma U/D-VDEs. This event is simulated by deliberately pushing the plasma up (or down), and then switching off the control system, as to mimic a loss of VS control, or a perturbation above the controller limits. This event is conservatively considered as "unmitigated", i.e. no mitigating actions are considered, to reduce part of the charged particle energy contribution by transforming it in radiation heat load, via shutter pellet injection (SPI) or massive gas injection (MGI) [14]. It is modeled in the following phases:

- First touch: The plasma moves (upward or downward) vertically and touches the wall. The most conservative case considered is that the plasma is in full power, i.e. in H-Mode, with an e-folding length $\lambda_{q}$ in the range of $1 \mathrm{~mm}$ (which is the DEMO prediction for the diverted configuration, before touching the wall) up to $5-6 \mathrm{~mm}$ (which is the prediction for outboard limiter configuration, hence after touching the wall), as described in [15].

- Thermal Quench: (TQ). When $q_{95 \%}=2$, representing the safety factor at $95 \%$ of the plasma boundary flux, the TQ is triggered. In this phase all thermal energy (1.3GJ, as in [15]), or half of it (the other half being lost in the pre-TQ), is released in $\approx 1-4 m s$. In this phase a broadening of the near SOL e-folding length of a factor $\approx 7[16]$ is considered (leading to $\lambda_{q} \approx 7 \mathrm{~mm}$ ), similarly to ITER [14], and the plasma current is increased by $5-10 \%$.

- Current Quench (CQ): The final phase is represented by the $\mathrm{CQ}$, when the plasma current decrease from $19 M A$ to 0 , in a time range, predicted for DEMO, from $74 \mathrm{~ms}$ to $200 \mathrm{~ms}$, as described in [10]. The decay of the plasma current during the CQ is assumed to be linear and, conservatively, the $85 \%$ of the magnetic energy is supposed to be converted into conducted energy, while the remaining $15 \%$ is radiated [17]. In this phase two values of e-folding length are used, i.e. $\lambda_{q}$ equal to $10 \mathrm{~mm}$ (conservative) and $30 \mathrm{~mm}$ (more realistic).

Controllable perturbations. These are the plasma perturbations which the controller is able to reject, avoiding plasma-wall contact, but decreasing the nominal clearance from the $\mathrm{FW}$, set to $22.5 \mathrm{~cm}$ at the outer midplane. These perturbations are taken into account in the design of the baseline plasma scenario parameters (e.g. maximum elongation, machine geometry), which is chosen in order to stay within the control limits, during such occurrences. Similarly to [4], the calculations of the plasma perturbation dynamic simulations are performed using the CREATE-NL 2D code, on the 2017 DEMO baseline [11], with simplified assumptions about the controller. A best achievable performance controller [18] was used to control the vertical position, i.e. a voltage was given as input to the system equal to 5-10 times the ideal value that would stop the plasma vertical movement at infinity. The disturbances are modelled as instantaneous 
variation of poloidal beta $\left(\beta_{p o l}\right)$ and internal inductance (li), for: a) ELMs, with $\Delta l i=0.1$, and $\Delta \beta_{p o l}=-0.1$, and b) minor disruptions, with $\Delta l i=-0.1$, and $\Delta \beta_{p o l}=-0.1$. These perturbations are considered to occur during the plasma flat top. The hypotheses used for the heat flux (HF) calculation are the same used in [4], i.e. $P_{\text {far }-S O L}=70 \mathrm{MW}$ and $\lambda_{q}=50 \mathrm{~mm}$, representing, respectively, the power crossing the separatrix entering in the channel relative to the charged particle blobby transport, and the corresponding e-folding length [19].

Plasma mitigated disruptions. Such events are preliminarily evaluated based mainly on the work performed for ITER in [14]. The main reason is to have an initial evaluation of the strategy to have discrete limiters. Those are effective in case of the heat flux associated with the charged particles, which follow the magnetic field lines, hence colliding with the PFCs with a shallow angle and in concentrated areas. They are ineffective in case of radiative heat flux, which are deliberately increased during a mitigated disruption. In this occurrence the radiation energy is spread uniformly across the FW and the limiters, the latter hence becoming ineffective in the protection of the former, but with a consequent overall lower average density. Starting from the U-VDE case, described above, the following parameters, extrapolated from [14], are used:

- Pre-TQ: $20 \%$ of the total thermal energy, i.e. $0.26 G J$ of the $1.3 G J[15]$, is released at the time where the material from SPI or MGI arrive to the plasma.

- Mitigated TQ: For a successful mitigation $80 \%$ (90\% is the target for ITER [20]) of the remaining $\approx 1 G J$ is radiated in $\approx 1-3 m s$. This deposition time depends from the gas mix used for the mitigation. This leads to a radiated power worst case $P_{\text {rad }} \approx$ $800 \mathrm{GW}$. A total toroidal and poloidal peaking factor TPF of 2.8 is used, similarly to ITER.

H-L transitions. These transients deal with various scenarios that may lead to the loss of plasma confinement during the flat-top, hence leading to an exit from the (presently considered) H-Mode scenario. A list of such events, described in [21], was computed using the ASTRA code [22], and provided in terms of time evolution of the main plasma internal parameters, i.e. $\beta_{\text {pol }}$ $l i$, and $I_{p}$, shown in Fig. 4 . These inputs were used to run several EM simulations, with the CREATE NL code, including the currents in the passive structures, with and ideal controllers and diagnostics, simplified power supply transfer functions, and poloidal filed coils voltage limits. The transients included are:

- Loss of auxiliary plasma heating power $\left(\Delta \beta_{p o l} \approx-0.4\right.$ in $4 s$ ).

- Tungsten event, tungsten impurity entering the plasma $\left(\Delta \beta_{\text {pol }} \approx-0.64\right.$ in $\left.4 s\right)$.

- Unexpected TQ on intermediate timescale $\left(\Delta \beta_{p o l} \approx\right.$ -1 in $3 s$ ). Event deliberately conservative in terms of $\Delta \beta_{p o l}$, to test the design of a possible limiter in the inner wall.
For all the perturbation considered, including a list of more recent ones, the controller was able to avoid the plasma wall contact, keeping a minimum distance of $5 \mathrm{~cm}$. It was decided nevertheless, to include the last conservative case, named "Unexpected TQ on intermediate timescale", when the $\Delta \beta_{p o l}$ variation is larger than the one evaluated by the ASTRA code, and fast enough, such as to be above the controller limits, leading the plasma to become in contact with the inboard wall (Fig. 5). The fact to use at this stage such conservative event is considered as a mitigation strategy because: 1) more perturbations may become available in the future, with larger variation than the one foreseen so far, and giving the natural tendency of the plasma to move inwards when suddenly loses energy, and 2) to take into account the possible detrimental effects that will be introduced by realistic controllers and diagnostics. Due to the large uncertainties regarding the state of the plasma when touching the inner wall the parameters used for the $\mathrm{HF}$ calculation are $P_{S O L}$ in the range $30-60 \mathrm{MW}$, and $\lambda_{q}=4 \mathrm{~mm}$ (in line with what is foreseen for the inboard limiter case in [15]).
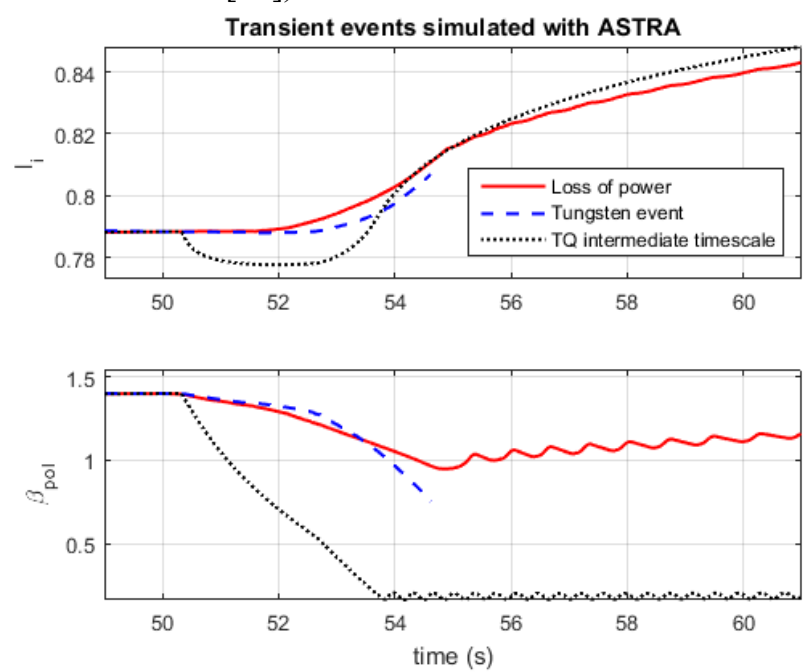

Fig. 4 List of some of the plasma perturbations amplitude and time evolution, as calculated by the ASTRA code, in terms of $\Delta \beta_{p o l}$ and $\Delta l i$, and used as inputs in the electromagnetic simulations. The TQ intermediate timescale is a conservative case deliberately chosen to be above controller limits.

\section{Poloidal location and shape design of first wall protection limiters}

The list of plasma transient simulations, described in paragraph 4, is used iteratively by evaluating the heat flux on the PFC, and giving a feedback to the scenario and machine optimization. A complete 3D FW design is used to evaluate the heat flux loads in all the plasma phases, including charged particles and radiative loads, in normal and off-normal cases. The magnetic flux-maps, generated by the EM simulations in the time instant of interest of the events, are used by the $3 \mathrm{D}$ field line tracing codes PFCflux [23] and SMARDDA [24], with the aim of: a) designing the limiters poloidal and toroidal shape and extension, to be able to protect the BB FW, b) design the surface shape and component geometry to distribute the heat flux as evenly as possible, and c) minimizing the 
limiter number and size to have the smallest possible impact on TBR and reduce cost/complexity.
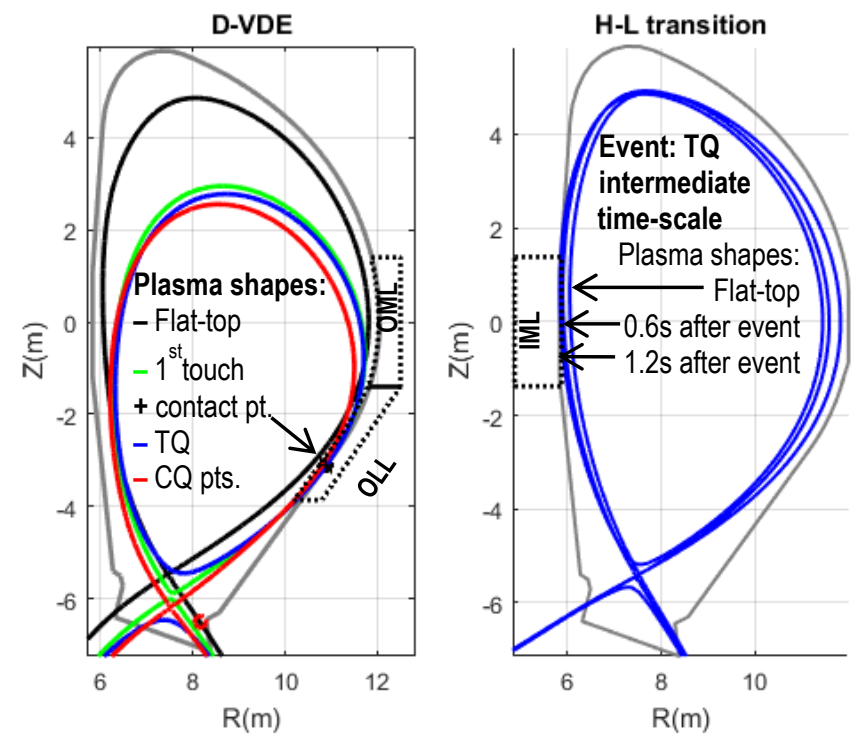

Fig. 5 Simulation of plasma off-normal event transients for: DVDE (left) and H-L transition (TQ intermediate timescale, conservative case, on the right).

At present, four poloidal positions have been analyzed to locate discrete protection limiters, with the aim to satisfy the criteria that the heat flux on the FW remains, under all considered circumstances, below the limit of $\approx$ $1-2 \mathrm{MW} / \mathrm{m}^{2}$ in steady state, while higher but fast transient cases will be analyzed case by case in the paragraph 6 . A list of the presently considered limiters, presented also in $[25,26]$ for what concern the integration engineering aspects, is shown in the Fig. 6, and hereafter reported:

- 4 Outboard Mid-plane Limiters (OML), designed for plasma ramp up and down.

- 8 Upper Limiters (UL), designed for U-VDE.

- 4 Outboard Lower Limiters (OLL), designed for D-VDE.

- 4 Inboard Mid-plane Limiters (IML), designed for H-L transitions, and in general for all the events characterized by a sudden loss of the plasma confinement energy.

The toroidal and poloidal extension of the OML and UL are restricted, respectively, by the maximum extension of the outboard equatorial port, described in [26], and the available space in the upper vertical port, described in [12]. The IML size is presently constrained also by the outboard equatorial port, from which a front maintenance scheme is preliminary being developed, and described in [25]. Finally the OLL limiter, recently added, is presently being developed and is thought to be maintained also by the equatorial port. In the Tab. 1 is reported a summary of all the steady state and plasma transient analyzed, described in the paragraph 4 . The critical cases are reported in bold and with a numbered superscript. The results indicate the maximum HF density on all the limiters, and FW. As described in [27], a consistency check was carried out for every case, by integrating the HF density on all the PFC surfaces, (including $\mathrm{FW}$, limiters and divertor) to verify that the resulting charged particles power is equal to the power injected at the outer mid-plane $\left(P_{S O L}\right)$, used in the 3D field-line tracing codes. A power ratio $\rho$ is introduced to describe the resulting integrated power, over the injected one:

$$
\rho=\frac{\int_{\text {surface }} H F d s}{P_{S O L}}
$$

The HF values, calculated by the codes, are then uniformly multiplied by the inverse of $\rho$, typically in the range $0.1(90 \%$ of power loss) to 1 (no power loss), as for ITER [28], in order to get the power ratio equal to 1.

The reported values show that the limiters are able to protect the BB FW, satisfying a HF limit below 1$2 \mathrm{MW} / \mathrm{m}^{2}$ for time durations of few seconds, or below few tens of $M W / \mathrm{m}^{2}$, typically in the TQ cases, but for very short time of the order of less than $10 \mathrm{~ms}$. The effects on the presently considered BB FW plasma facing component are shown in the paragraph 6 .

The results, reported in the table, for the limiters show that, by design, different locations have to deal with different transients, typically from the order of less than ten of $M W / \mathrm{m}^{2}$ for tens of $s$, which can be achieved by using a technology similar to the present DEMO divertor [29], up to tens or hundreds of $G W / m^{2}$ for few $m s$. Different preliminary potential design solutions are proposed in paragraph 6 for the different transients and functions of the limiters. An overall picture of the present design of the various limiters is show in Fig. 6, where the maximum heat fluxes are shown for each limiter. Meanwhile sensitivity analyses are being carried out to evaluate the impact of limiters and FW panel misalignment, with respect to the nominal position, and are described in $[30,31]$.

Finally, the last case in the table represents the mitigated disruption, run using the CHERAB code [32]. This event is preliminary run to calculate whether the radiation energy HF on all the surfaces facing the plasma is above the BB FW limits for the case of an ideally mitigated disruption. In this case the radiation energy spreads uniformly on the FW and the limiters, the latter becoming ineffective in protecting the former. Preliminary results were obtained by equally dividing the radiation power of $800 G W$, derived in paragraph 4.2, amongst few hundreds of source points placed on the plasma boundary of the U-VDE TQ case. The calculation of the radiated energy resulted in an HF on the limiters and the FW equal to 1 to $3 \mathrm{GW} / \mathrm{m}^{2}$ in case of a deposition time equal to, respectively, $3 m s$ or $1 m s$. This HF, although very brief, may prove to be challenging for any PFC armor surface, including the $\mathrm{FW}$, as shown in the paragraph 6 . As a result it may be necessary to prescribe, to the disruption mitigation system, a lower radiation HF fraction, to avoid damages to the FW, leaving an higher fraction to the charged particles that will impact on the limiters. Both charged particles and radiation heat loads are produced on the same $3 \mathrm{D}$ mesh, which is used to run 3D thermo-hydraulic calculations of FW and limiter [33] designs. 


\section{Simplified thermo-hydraulic analysis on DEMO PFC during plasma transients}

Starting from the HF results shown in Tab. 1 the RACLETTE code [34] was employed to evaluate the thermal response of the PFCs and to optimize the PFC design of the different limiters. The attention is focused on 3 different designs, also described in [4], while the poloidal location is shown in Fig. 6:

1. First Wall (FW): $2 \mathrm{~mm}$ Tungsten (W) armor, $2 \mathrm{~mm}$ EUROFER heat sink, He coolant at $\approx 400^{\circ} \mathrm{C}, 80 \mathrm{~m} / \mathrm{s}$ velocity or water coolant at $\approx 300^{\circ} \mathrm{C}$ and $8 \mathrm{~m} / \mathrm{s}$ velocity. These represent the present BB PFC designs, able to withstand up to $1-2 \mathrm{MW} / \mathrm{m}^{2}$ [2].

2. Divertor like (Div-like) limiter: $8 \mathrm{~mm}$ Tungsten (W) armor, $2 \mathrm{~mm}$ Copper alloy (CuCrZr) heat sink, water as coolant at $\approx 150^{\circ} \mathrm{C}$ temperature, and $8 \mathrm{~m} / \mathrm{s}$ velocity. This design can withstand steady state of $10 \mathrm{MW} / \mathrm{m}^{2}$, and hundreds of transients of tens of seconds and up to $20 \mathrm{MW} / \mathrm{m}^{2}$ [29]. For this reason this solution is proposed and analyzed for the OML, which are designed to deal with normal events.

3. Sacrificial Limiter (SL): The same parameters as the divertor-like limiter, but with $20 \mathrm{~mm}$ Tungsten (W) armor. This design aims at taking advantage of the increased thermal capacity and reduced thermal conductivity of the thick $\mathrm{W}$, and is meant to deal with very high HFs $\backslash$ (tens to hundreds of $G W / m^{2}$ ), for very short time $\leq 10 \mathrm{~ms}$. In such conditions typically only the limiter tungsten armor surface experiences a strong variation of temperature, which may reach the melting value of $3422^{\circ} \mathrm{C}$, while the materials below, depending on the total energy, remain almost unaffected. This design is proposed and analyzed for the UL, OLL and IML, which are designed to deal with off-normal events.

In Tab. 2 the results of the RACLETTE code are reported for the critical cases extracted from the Tab. 1. The calculations are done by considering the max power density, reported in the "max HF" column of Tab. 1, and maintaining it constant for the longest deposition time indicated, to be conservative, and starting from a steady state HF of $1 \mathrm{MW} / \mathrm{m}^{2}$ on all PFCs. In the columns of Tab. 2 are reported the case number, the $\mathrm{W}$-armor evaporation and melting thickness (in $\mu \mathrm{m}$ ), and the armor surface (surf.) and heat sink temperature (in ${ }^{\circ} \mathrm{C}$ ).

For the cases regarding the FW from ${ }^{(1)}$ to ${ }^{(3)}$, the deposition time is too short to lead either to any $\mathrm{W}$-armor melting or evaporation, or to the EUROFER heatsink going above $550^{\circ} \mathrm{C}$, chosen as a conservative criteria based on [35]. In the case (4) instead, representing an ideally mitigated disruption $\mathrm{TQ}$, up to $38 \mu \mathrm{m}$ of the Warmor are molten. As mentioned in the last section of paragraph 5, it is necessary to prescribe a lower radiation fraction for the mitigation system, comparing to the ITER target value of $80-90 \%$, considered also in this DEMO case. This may require either a strategy to ensure a reduction of the energy available at the TQ, or to reduce the fraction going in the radiation.

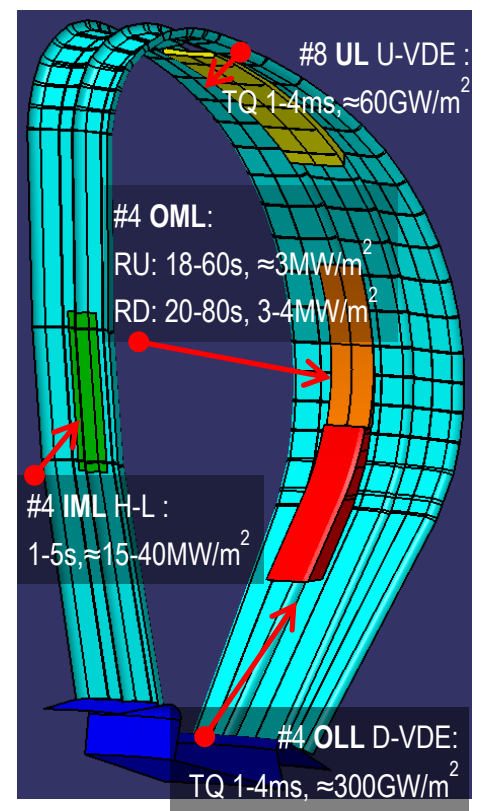

Fig. 6. Present limiters 3D surface design. For each of them is reported the minimum number (\#) needed and the worst case unmitigated $\mathrm{HF}$ and deposition time they need to withstand.

In the sacrificial limiter cases, severe damages occur to the armor surface, both in terms of melting, and evaporation, in the most severe cases. Nevertheless, due to the presence of the $20 \mathrm{~mm}$ thick $\mathrm{W}$-armor, the $\mathrm{CuCrZr}$ pipe remains in all cases below its temperature limit of $350^{\circ} \mathrm{C}$, chosen as a conservative criteria based on [36], hence preserving the integrity of the limiters cooling structure. In the case ${ }^{(7)}$, representing the first touch of the $\mathrm{D}$-VDE, up to $\approx 700 \mu \mathrm{m}$ of the OLL limiter $\mathrm{W}$-armor is molten, and up to $82 \mu m$ is evaporated. Similarly in the case ${ }^{(10)}$, representing the plasma-wall contact on the IML after an $\mathrm{H}-\mathrm{L}$ transition, up to $120 \mu \mathrm{m}$ of $\mathrm{W}$-armor is evaporated, and up to $\approx 3.5 \mathrm{~mm}$ is molten. At present, the RACLETTE code deals with the vaporization in such a way that the vaporized material, and the energy needed to vaporize it, disappear instantaneously, neglecting possible vapor shielding effects, which may reduce the incoming HF to the surface. For this reason, all the cases with vaporization larger than few tens of $\mu m$ should be considered as a worst case. More sophisticated codes, such as TOKES, have been employed to evaluate the vapor shielding effects in ITER [32] and DEMO [33]. More extreme cases are ${ }^{(6)}$ and ${ }^{(9)}$, representing the TQ of unmitigated $U$ and D-VDEs, respectively. In these cases up to $\approx 2.5 \mathrm{~mm}$ of $\mathrm{W}$-armor are predicted to evaporate by applying $58.8 \mathrm{GW} / \mathrm{m}^{2}$ for $4 \mathrm{~ms}$, for the first case, while in the second case was not possible to obtain a convergence of the code. Also for these cases the present RACLETTE code is not adequate to simulate the extreme real simulation, and they should be considered as conservative results.

For these extreme cases an R\&D activity is being proposed, within the DEMO divertor work package (WPDIV), to develop tungsten materials [37] with similar thermal capacity of bulk tungsten, but lower thermal conductivity, which could help to reduce the HF to the coolant pipe. The testing of such samples is also being planned on experimental devices. Finally, the case (4) 
represents again an ideally mitigated disruption, where the radiation HF is uniformly spread on all the surfaces facing the plasma. As for the FW, and similarly to what is also found for the divertor-like design, few tens of $\mu m$ of the
$\mathrm{W}$-armor are molten, resulting in a damage of the PFC surface. It is confirmed that a fine tuning of the mitigation system requirements may be needed to avoid such damages.

\begin{tabular}{|c|c|c|c|c|c|c|}
\hline \multicolumn{4}{|c|}{ 3D field-line tracing Inputs for charged particle HF } & & \multicolumn{2}{|c|}{ - Outputs: $\max$ HF $\left(\mathrm{MW} / \mathrm{m}^{2}\right)$} \\
\hline $\begin{array}{l}\text { Scenario (main } \\
\text { limiter) }\end{array}$ & Case(s) & $\mathrm{P}_{\mathrm{SOL}}(\mathrm{MW})$ & $\lambda_{\mathrm{q}}(\mathrm{mm})$ & $\begin{array}{c}\text { Deposition } \\
\text { time (s) }\end{array}$ & $\begin{array}{l}\text { On limiters ( } \rho- \\
\text { rescaled) }\end{array}$ & $\begin{array}{l}\text { On FW ( } \rho- \\
\text { rescaled) }\end{array}$ \\
\hline SOF/EOF (all) & Diverted & 69 & 50 & Steady state & 4.29 & 1.88 \\
\hline $\begin{array}{c}\text { Min. disr. \& ELM } \\
\text { (all) }\end{array}$ & Diverted & 69 & 50 & $15-50 \mathrm{~ms}$ & 1.31 & $4.83^{(1)}$ \\
\hline Ramp-Up (OML) & Limited & 3.5 & 6 & $17.5-35$ & 2.40 & 0.32 \\
\hline \multirow{4}{*}{$\begin{array}{l}\text { Ramp-Down } \\
\text { (OML) }\end{array}$} & \multirow{4}{*}{ Limited } & \multirow{2}{*}{5} & 6 & $25-50$ & 3.61 & 0 \\
\hline & & & 50 & $25-50$ & 4.51 & 0.47 \\
\hline & & \multirow{2}{*}{7.5} & 6 & $37.5-75$ & 4.19 & 0 \\
\hline & & & 50 & $37.5-75$ & 3.14 & 0.42 \\
\hline \multirow{5}{*}{ U-VDE (UL) } & \multirow{2}{*}{$\begin{array}{l}\text { First } \\
\text { touch }\end{array}$} & \multirow{2}{*}{69} & 1 & $20-35 \mathrm{~ms}$ & 100 (5) & $\overline{0}$ \\
\hline & & & 5 & $20-35 \mathrm{~ms}$ & 15.9 & 0.04 \\
\hline & TQ & $325 \mathrm{GW}$ & 7 & $1-4 \mathrm{~ms}$ & $\mathbf{5 8 , 8 0 0}(\underline{6})$ & $286^{(2)}$ \\
\hline & \multirow{2}{*}{$\begin{array}{l}\text { CQ1 \& } \\
\text { CQ2 }\end{array}$} & \multirow{2}{*}{10} & 10 & $74-200 \mathrm{~ms}$ & 4.68 & 0.05 \\
\hline & & & 30 & $74-200 \mathrm{~ms}$ & 6.07 & 0.22 \\
\hline \multirow{5}{*}{ D-VDE (OLL) } & \multirow{2}{*}{$\begin{array}{l}\text { First } \\
\text { touch }\end{array}$} & \multirow{2}{*}{69} & 1 & $15-35 \mathrm{~ms}$ & $623^{(7)}$ & $\overline{0}$ \\
\hline & & & 5 & $15-35 \mathrm{~ms}$ & $51.8^{(8)}$ & 0 \\
\hline & TQ & $325 \mathrm{GW}$ & 7 & $1-4 \mathrm{~ms}$ & 300,000 (9) & $5.9^{(3)}$ \\
\hline & \multirow{2}{*}{$\begin{array}{l}\text { CQ1 \& } \\
\text { CQ2 }\end{array}$} & \multirow{2}{*}{10} & 10 & $74-200 \mathrm{~ms}$ & 10.8 & 0 \\
\hline & & & 30 & $74-200 \mathrm{~ms}$ & 19.2 & 0.14 \\
\hline \multirow{2}{*}{$\begin{array}{l}\text { H-L transition } \\
\text { (IML) }\end{array}$} & \multirow{2}{*}{$\begin{array}{l}\text { Limited } \\
\text { (inboard) }\end{array}$} & \multirow{2}{*}{30} & 2 & $1-5$ & $39.56(10)$ & 0.12 \\
\hline & & & 4 & $1-5$ & 14.78 & 1.81 \\
\hline \multicolumn{4}{|c|}{ 3D Inputs for radiation HF (CHERAB) } & & \multicolumn{2}{|c|}{ Outputs: max HF $\left(\mathrm{MW} / \mathrm{m}^{2}\right)$} \\
\hline & Case & \multicolumn{2}{|c|}{$\mathrm{P}_{\mathrm{SOL}}(\mathrm{MW})$} & $\begin{array}{l}\text { Deposition } \\
\text { time (s) }\end{array}$ & \multicolumn{2}{|c|}{ On limiters \& FT, with TPF 2.8} \\
\hline $\begin{array}{c}\text { Mitigated } \\
\text { disruption (all) }\end{array}$ & $\begin{array}{l}\text { Mitig. - } \\
\text { TQ }\end{array}$ & \multicolumn{2}{|c|}{$800 \mathrm{GW}$} & $1-3 \mathrm{~ms}$ & \multicolumn{2}{|c|}{$\approx 3,000-1,000$ (4) } \\
\hline
\end{tabular}

Tab. 1 Summary of all the plasma transient cases analyzed. The results report the HF due to charged particles, evaluated with 3D field-line tracing codes, on the designed FW and limiters geometry and number. The subset of critical cases in highlighted in bold, indicated with a superscript number within brackets.

\section{Conclusions and future work}

In this paper it is presented the proposed strategy for the protection of the EU-DEMO BB FW from plasma transients. Discrete high heat flux limiters are proposed to protect the FW, with different designs, according to the expected heat flux they will receive. Two different cases are foreseen: 1) DEMO divertor-like limiters, capable of exhausting up to few tens of $M W / \mathrm{m}^{2}$ for few tens of $s$, or 2) sacrificial limiters with thicker armor, up to $20 \mathrm{~mm}$, able to sustain from hundreds of $M W / \mathrm{m}^{2}$ to tens of $\mathrm{GW} / \mathrm{m}^{2}$ for times up to few tens of $m s$. The sacrificial limiters are designed aiming at keeping the integrity of their cooling system, although they may experience damage to their armor surface. A list of plasma perturbations is used to run EM simulations, using CREATE-NL [5] and MAXFEA [6] 2D codes, and CARMA0NL 3D code [7], with the aim to predict all the possible plasma-wall contact locations. The PFCflux [23] and SMARDDA [24] codes are used to evaluate the heat flux of the PFCs due to charged particles loads, while the CHERAB [32] code is used for the radiation loads. These loads are used to run simplified thermal analysis on the different PFCs, and 
propose design variations using the RACLETTE [34] code. Using an iterative procedure, with a feedback on plasma and geometry optimization, the minimum number of limiters able to prevent damages to the FW in all the considered transients is proposed. The results show that it is possible to prevent failure of the $\mathrm{BB} F W$ cooling system, and even any damage to the FW system for any of the listed cases. To stay within the limits also in the case of an ideally mitigated disruption, a constraint needs to be prescribed on the maximum radiation obtained by any possible mitigation system, and/or increasing the loss of energy in the pre-thermal quench. Additional limiter locations, not presented in this paper, are also being explored in parallel as a mitigation strategy for events not yet considered. This is important to analyze their possible maintainability, above all for areas far from ports, such as on the inboard side of the upper port, which will likely require front maintenance schemes.

First Wall (EUROFER heat sink temp. limit $550^{\circ} \mathrm{C}$ )

\begin{tabular}{|c||c|c|c|c|}
\hline Case & $\begin{array}{c}\text { W-Evap. } \\
(\mu \mathrm{m})\end{array}$ & $\begin{array}{c}\text { W-Melt. } \\
(\mu \mathrm{m})\end{array}$ & $\begin{array}{c}\text { Surf. temp. } \\
\left({ }^{\circ} \mathrm{C}\right)\end{array}$ & $\begin{array}{c}\text { Heat sink } \\
\text { temp. }\left({ }^{\circ} \mathrm{C}\right)\end{array}$ \\
\cline { 2 - 5 }$(\underline{\mathbf{1}})$ & 0 & 0 & 465 & 394 \\
\hline$\underline{(2)}$ & 0 & 0 & 1545 & 448 \\
\hline$\underline{(3)}$ & 0 & 0 & 432 & 384 \\
\hline \multirow{2}{(4)}{} & 0 & $\mathbf{3 8}$ & $\mathbf{3 8 7 2}$ & 545 \\
\hline \multirow{2}{*}{} & &
\end{tabular}

Sacrificial limiter: (CuCrZr heat sink temp. $\left.\lim .350^{\circ} \mathrm{C}\right)$

\begin{tabular}{|c|c|c|c|c|}
\hline (4) & 0 & 38 & 3879 & 172 \\
\hline (5) & 0 & 0 & 1500 & 171 \\
\hline (6) & 2560 & 988 & 7867 & 176 \\
\hline (7) & 82 & 698 & 5470 & 179 \\
\hline (8) & 0 & 0 & 922 & 171 \\
\hline (9) & \multicolumn{4}{|c|}{ Not converged } \\
\hline$\underline{(10)}$ & 120 & 3470 & 4408 & 280 \\
\hline \multicolumn{5}{|c|}{ Divertor like limiter: $\left(\mathrm{CuCrZr}\right.$ heat sink temp. $\left.\lim .350^{\circ} \mathrm{C}\right)$} \\
\hline (4) & 0 & 23 & 3695 & 184 \\
\hline
\end{tabular}

Tab. 2 Results of simplified thermal analysis, run with the RACLETTE code, on different HF cases and PFC designs. Results reported in bold indicate values above the chosen material limits.

It is possible to prevent damages also for the limiters with divertor-like PFCs, proposed for the plasma rampup/down phases, which are normal transients that will happen at the beginning and end of every DEMO pulse.

Finally it seems also possible to prevent the failure of the proposed sacrificial limiters cooling systems, in case of off-normal events, although in the most extreme cases, e.g. during unmitigated disruption TQ, severe damages are expected on the sacrificial limiters armor surface, up to $\approx 3.5 \mathrm{~mm}$ deep, of the total $20 \mathrm{~mm}$ of the design proposed. The preliminary simulations do not include the possible mitigation effects coming from the vapor shielding, which is calculated with dedicated codes, for instance, both for ITER [38] and DEMO [39], and hence has to be intended conservative. Studies on the damages due to runaway electrons (REs) [40] are also being carried out in DEMO to evaluate the integrity of the limiters, the FW and their coolant also during the plasma disruptions. Such events have to be absolutely minimized, and for this reason as stable as possible plasma scenarios are being investigated for DEMO [13]. The present strategy is to preferentially place sacrificial limiters as far as possible from the plasma, in order to be engaged by it only in the rare off- normal events. The idea is that, by doing so, even a limiter with a slightly damaged surface, for instance from a previous event, may still maintain its function to protect the FW. Modelling and experimental studies will be performed in the future to evaluate if different limiter designs, also with advanced materials, as described in [37], can withstand more than one event.

\section{Acknowledgments}

This work has been carried out within the framework of the EUROfusion Consortium and has received funding from the Euratom research and training programme 20142018 and 2019-2020 under grant agreement No 633053. The views and opinions expressed herein do not necessarily reflect those of the European Commission.

\section{References}

[1] C. Bachmann, et al., "This conference".

[2] F. Cismondi, et al., "Progress in EU Breeding Blanket design and integration," FED, vol. 136, pp. 782-792, 2018.

[3] F. Maviglia, et al., "Effect of engineering constraints on charged particle wall heat loads in DEMO," FED, vol. 124, pp. 385-390, 2017.

[4] F.Maviglia, et al., "Wall protection strategies for DEMO plasma transients," FED, vol. 136, pp. 410-414, 2018.

[5] R.Albanese, et al., "CREATE-NL+: A robust controloriented free boundary dynamic plasma equilibrium solver," FED, vol. 96-97, pp. 664-667, 2015.

[6] P. Barabaschi, "The MAXFEA code," in ITER EDA Plasma Control Technical Meeting, Naka, Japan, 1993.

[7] F. Villone, et al., "Coupling of nonlinear axisymmetric plasma evolution with three-dimensional volumetric conductors," PPCF, vol. 55 , p. 095008, 2013.

[8] F. Villone, et al., "Production of 3D linearized models (Internal Report)," 2015.

[9] R. Albanese, et al., "Vertical Stability (VS) Analysis (Internal report 2N6C89)," 2018.

[10] F. Maviglia, et al., "Optimization of DEMO geometry and disruption location prediction," FED, vol. 146, pp. 967971, 2019.

[11] G. Federici, et al., "DEMO design activity in Europe: Progress and updates," FED, vol. 136, pp. 729-741, 2018.

[12] C. Vorpahl, et al., "Initial configuration studies of the upper vertical port of the European DEMO," FED, 2019 (In Press).

[13] M. Siccinio, et al., "This conference".

[14] R. Pitts, et al., "Heat and Nuclear Load Specifications," (ITER internal report v2.3, idm_2LULDH).

[15] R. Wenninger, et al., "The DEMO wall load challenge," Nucl. Fusion, vol. 57, p. 046002, 2017.

[16] T. Hender, "Final report on DEMO disruption consultancy (Internal report)".

[17] G. Maddaluno, et al., "Wall loads during disruptions (EUROfusion internal report)," 2018.

[18] R. Albanese, et al., "Electromagnetic analyses of single and double null configurations in DEMO device," FED, 2019 (In Press).

[19] D. Carralero, et al., "Experimental validation of a filament transport model inturbulent magnetized plasmas," $P R L$, vol. 115, p. 215002, 2015.

[20] S. Putvinski, et al., "Physics design requirements for DMS," ITER internal report (ITER_D_6XTD3L), 2012. 
[21] E. Fable, "Report on E. Fable work on various transport related investigations for DEMO," (Internal Report idm_2LMNFR), 2017.

[22] G.V. Pereverzev, et al., "ASTRA - Automated System for TRansport Analysis," Max-Planck-Institut Für Plasmaphysik, IPP-Report, IPP 5/98, 2002.

[23] M. Firdaouss, et al., "Modelling of power deposition on the JET ITER like wall using the code PFCFlux," J. Nucl. Mater., vol. 348, p. 536, 2013.

[24] W. Arter, et al., "The SMARDDA approach to ray-tracing and particle tracking," IEEE Trans. Plasma Sci., Vols. 439, p. 3323, 2015.

[25] Z. Vizvary, et al., "European DEMO first wall shaping and limiters design and analysis status," This conference.

[26] T. Franke, et al.,, "The EU DEMO equatorial outboard limiter - design and port integration concept," This conference.

[27] J. Gerardin, et al., "Simplified heat load modeling for design of DEMO discrete limiter," Nuclear Materials and Energy, vol. 20, p. 100568, 2019.

[28] R. Mitteau, Private communications.

[29] J.H You, et al., "European divertor target concepts for DEMO: Design rationales and high heat flux performance," Nuclear Materials and Energy, vol. 16 , p. $1-11,2018$.

[30] M. L. Richiusa, et al., "DEMO Single Module Segment Concept First Wall and limiter misalignment study by 3D field line tracing," in This conference..

[31] e. a. Z.Vizvary, "DEMO First Wall misalignment study," FED (In PRess).

[32] M. Carr, et al., "Towards integrated data analysis of divertor diagnostics with ray-tracing," in 44th EPS Conference on Plasma Physics, 2017.

[33] T.R. Barrett, et al., "Designs and technologies for plasmafacing wall protection in EU DEMO," Nuc. Fus., vol. 59, 2019.

[34] A.R. Raffray, et al., "RACLETTE: A model for evaluating the thermal response of plasma facing components to slow high power plasma transients. Part I: Theory and description of model capabilities," Journal of Nuclear Materials, vol. 244, pp. 85-100, 1997.

[35] F. Arbeiter, et al., "Thermal-hydraulics of helium cooled First Wall channels and scoping investigations on performance improvement by application of ribs and mixing devices," FED, Vols. 109-111, pp. 1123-1129, 2016.

[36] M. Miskiewicz, et al., "Impact of plastic softening of overaged $\mathrm{CuCrZr}$ alloyheat sink tube on the structural reliability of a plasma-facing component," $F E D$, vol. 83 , p. 66-71, 2008.

[37] R.De Luca, et al., "Preliminary investigation on W foams as protection strategy for advanced FW PFCs," FED, 2019 (In Press).

[38] S. Pestchanyi, et al., "Simulation of divertor targets shielding during transients in ITER," FED, Vols. 109111, pp. 141-145, 2016.

[39] S. Pestchanyi, et al., "Simulation of the divertor target shielding during major disruption in DEMO," Fusion Science and Technology, In Press.

[40] F. Subba, et al., "Engineering analysis of the hot-spots due to runaway electrons in the EU-DEMO tungsten divertor," This conference. 